olfactory space. Distances between odours in this perceptual space correlated with physiological distances - a measure of the similarity of odours based on neural activation patterns - obtained in optical imaging experiments of antennal lobe activity.

This study confirms that olfactory neural activity corresponds to olfactory perception, and shows how invertebrate models such as the honeybee can be used to answer fundamental questions about the neurobiology of perception. As this line of enquiry is pursued with more odours, comprising a range of molecular features, we will gain a more complete description of the honeybee's olfactory perceptual space, and a better understanding of how perceptual measures relate to patterns of neural activity.

\section{Rebecca Craven}

\section{(10) References and links} ORIGINAL RESEARCH PAPER Guerrieri, F. et al. Perceptual and neural olfactory similarity in honeybees. PLoS Biol. 3, e60 (2005) FURTHER READING Sachse, S. et al. The spatial representation of chemical structures in the antennal lobe of honeybees: steps towards the olfactory code. Eur. J. Neurosci. 11, 3970-3982 (1999)

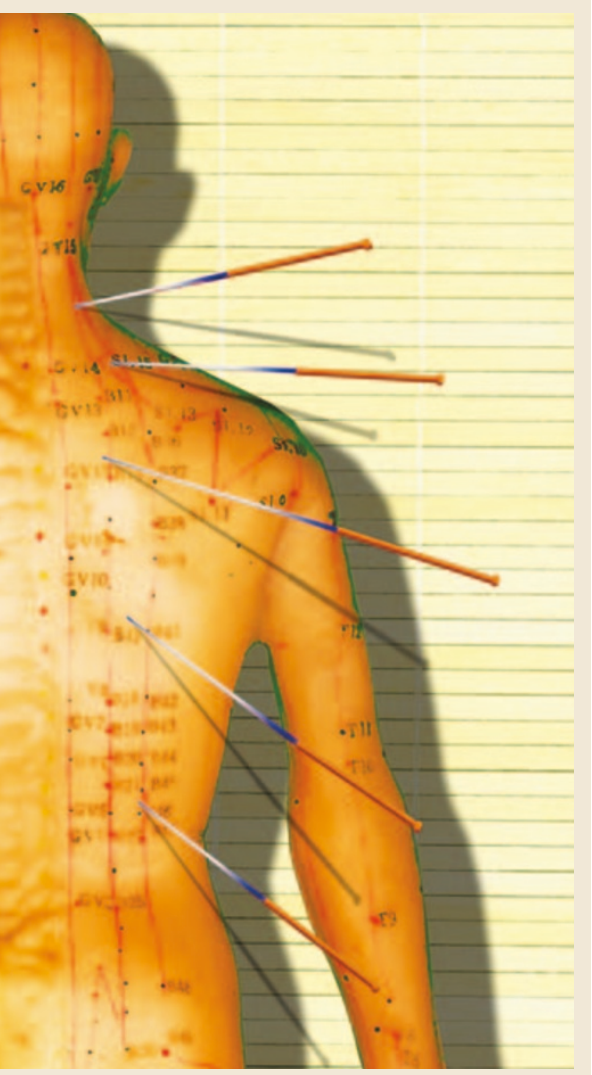

\title{
DEVELOPMENT
}

\section{MicroRNAs and brain morphogenesis}

Hundreds of microRNAs (miRNAs) have been identified in a range of organisms, from plants to fish to humans. These small, non-coding RNAs function as negative regulators of gene expression at the post-transcriptional level. Now, Schier and colleagues have used a zebrafish model to bring to light an important role for miRNAs in brain development.

The development of precurser miRNAs into mature miRNAs involves sequential cleavage events that are catalysed by two ribonuclease III (RNaseIII) enzymes, Drosha and Dicer. One strand of the resulting processed duplex is incorporated into a silencing complex, which acts on its target through either translational inhibition or mRNA cleavage.

Schier and colleagues generated dicer mutant zebrafish in which this process was disrupted and mature miRNAs were absent. In early-developing zygotes, maternally-derived Dicer is still active, so mature miRNAs are generated even if the zygotic dicer is mutated. The authors therefore used a germ line-replacement technique to abolish the maternal and zygotic functions of dicer in zebrafish.

In these mutant zebrafish, anterior-posterior and dorsal-ventral neural patterning occurred normally, and all principal subregions and cell types were present. This indicates that early patterning and fate specification were unaffected by the absence of miRNAs. However, there were major disruptions to morphogenesis during gastrulation, somitogenesis, and heart and brain development. The mutant zebrafish also developed at a reduced speed compared with their wild-type counterparts.

Specifically, abnormalities in neural development involved impairments in the positioning of neurons, in the defasciculation of longitudinally projecting axons and in touchinduced escape behaviour. Most strikingly, although the neural plate gave rise to a normal neural rod, the formation of the neural tube and neurocoel was disrupted. Normal ventricles did not form, and some boundaries that subdivide the brain into cell-tight compartments, such as the midbrain-hindbrain boundary, were missing. There were also defects in retinal development. So, miRNAs seem to be crucial for normal brain morphogenesis, neural differentiation and neural function during zebrafish development.

Further investigations revealed that the miR430 family of miRNAs is ubiquitously expressed and especially active during early stages of development. Importantly, injection of miR-430

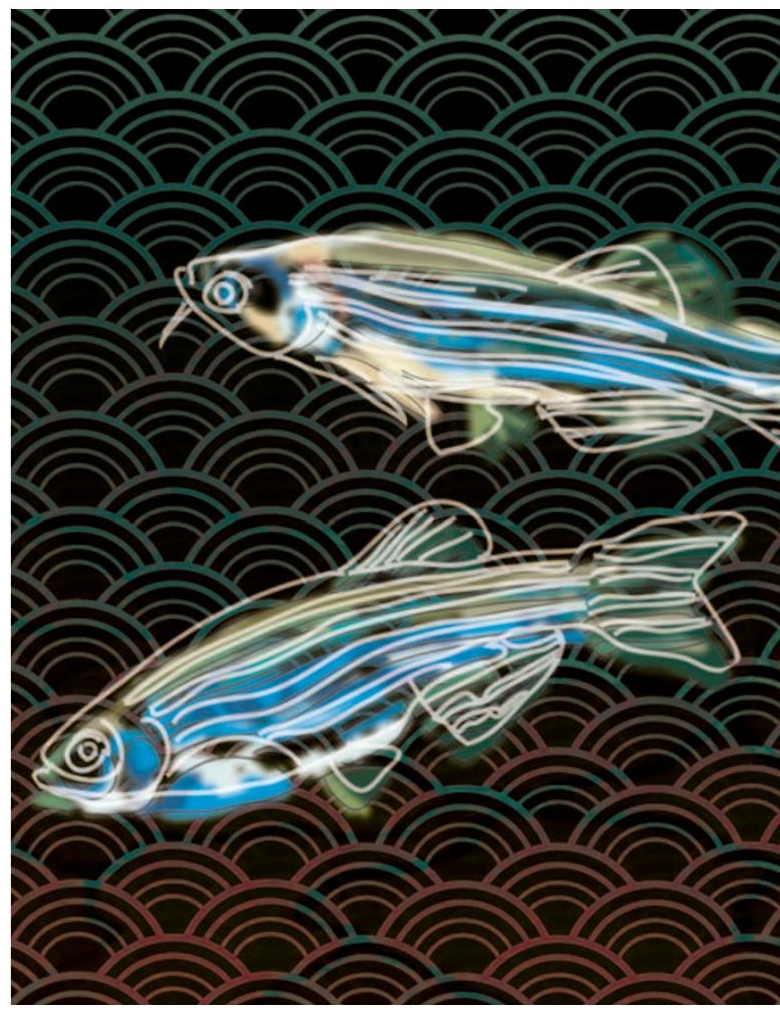

miRNAs into the mutant zebrafish rescued specific abnormalities in brain development, including the ventricle and boundary formation defects, although it had no effect on the heart and circulation abnormalities. This finding indicates that mature miRNAs are crucial for normal development and that the miR-430 family of miRNAs is responsible for the morphogenic defects seen in this study.

This work provides an important step in our understanding of the roles of miRNAs in brain development. More detailed knowledge of the molecular mechanisms that underlie brain morphogenesis - including neural tube and ventricle formation - could be gained through the study of the miR-430 miRNA family and its downstream targets. Moreover, the method used in this study - to prevent the generation of mature miRNAs in zebrafish - is just as noteworthy as the results, and could prove invaluable in future studies of the role of miRNAs in development.

Alison Rowan

\section{(2) References and links}

ORIGINAL RESEARCh PAPER Giraldez, A. J. et al. MicroRNAs

regulate brain morphogenesis in zebrafish. Science $\mathbf{3 0 8}, 833-838$ (2005)

FURTHER READING He, L. \& Hannon, G. J. MicroRNAs: small RNAs with a big role in gene regulation. Nature Rev. Genet. 5, 522-531 (2004) 